\title{
Palisada perforata (Rhodomelaceae, Ceramiales) en el Caribe mexicano
}

\author{
Palisada perforata (Rhodomelaceae, Ceramiales) from the Mexican Caribbean
}

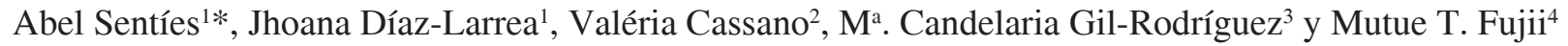 \\ ${ }^{1}$ Departamento de Hidrobiología, Universidad Autónoma Metropolitana-Iztapalapa. Apartado postal 55-535, 09340 México, D. F., México. \\ ${ }^{2}$ Departamento de Biologia Vegetal, Universidade do Estado do Rio de Janeiro. Maracanã, 20550-013 RJ, Brasil. \\ ${ }^{3}$ Departamento de Biología Vegetal (Botánica), Universidad de La Laguna. 38071 La Laguna, Tenerife, España. \\ ${ }^{4}$ Instituto de Botânica, Seção Ficologia. Caixa postal 3005, 01061-970 São Paulo, Brasil. \\ *Correspondencia: asg@xanum.uam.mx
}

\begin{abstract}
Resumen. Palisada perforata fue originalmente descrita para el litoral de Tenerife (islas Canarias, España), está ampliamente distribuida en el océano Atlántico oriental en la zona tropical y subtropical, mientras que en el lado occidental sólo está registrada como dudosa en Venezuela y Brasil. Además de las características típicas del género, esta especie presenta un crecimiento cespitoso con ejes decumbentes y arqueados y ramificación unilateral. En el presente trabajo los autores aportan por primera vez una descripción concisa de las estructuras vegetativas y tetraspóricas y se amplía la distribución geográfica de P. perforata con un nuevo registro en el Caribe mexicano.
\end{abstract}

Palabras clave: Palisada, morfología, Laurencia sensu lato, Rhodophyta, taxonomía.

\begin{abstract}
Palisada perforata was originally described from Tenerife (Canary Islands, Spain). This species has a wide distribution in tropical and subtropical zones of the Eastern Atlantic Ocean, but in the Western Atlantic there is only a doubtful record for Venezuela and Brazil. This species presents all typical features of the genus Palisada, being characterized by turf-like growth, the presence of arcuate and decumbent branches with erect branches disposed unilaterally. In the present paper we provide a detailed description of the vegetative and tetrasporangial structures. The geographical distribution of $P$. perforata in the Western Atlantic ocean was amplified with a new record in the Mexican Caribbean.
\end{abstract}

Key words: Palisada, morphology, Laurencia sensu lato, Rhodophyta, taxonomy.

\section{Introducción}

En las últimas 2 décadas el sistema de clasificación del complejo Laurencia J.V. Lamouroux ha tenido cambios significativos, entre los que destacan la resurrección del género Osmundea Stackhouse (Nam et al., 1994); la elevación del subgénero Chondrophycus Tokida et Saito in Saito (1967) a nivel genérico (Garbary y Harper, 1998); el establecimiento reciente del género Palisada K.W. Nam (Nam, 2007);, modificaciones nomenclaturales, registros nuevos y la creación de nuevas especies (Wynne y Ballantine, 1991; Gil-Rodríguez y Haroun, 1992; CordeiroMarino et al., 1994; Ballantine y Aponte, 1995; Fujii y Cordeiro-Marino, 1996; Furnari et al., 2002; Cassano et al., 2006; Fujii et al., 2006, entre otros).

En la actualidad, el complejo Laurencia lo integran los géneros: Laurencia sensu stricto J.V. Lamouroux, Osmundea, Chondrophycus y Palisada. Las diferencias

Recibido: 14 septiembre 2007; aceptado: 21 octubre 2008 básicas entre ellos, son: el número de células pericentrales por cada segmento axial vegetativo, el origen de los tetrasporángios, la posición de la primera célula pericentral en relación al tricoblasto, presencia y ausencia de fertilidad de la segunda célula pericentral, número de células pericentrales estériles en el segmento tetrasporangial, el origen y tipo de ramas espermatangiales y el número de células pericentrales en el segmento que origina el procarpo (Nam et al., 1994; Nam, 1999, 2006, 2007; Furnari et al., 2001).

Alrededor de 15 especies de Laurencia sensu lato han sido registradas en el Caribe mexicano (Sentíes y Fujii, 2002); no obstante, en esta región sólo L. venusta Yamada (Sentíes et al., 2001), Chondrophycus gemmiferus (Harvey) Garbary et J. Harper y C. poiteaui (J.V.Lamour.) K.W. Nam (comoLaurencia gemmifera y L. poiteaui, respectivamente; Fujii et al.,1996) han sido caracterizadas adecuada y ampliamente en términos morfológicos. Recientemente, Díaz-Larrea et al. (2007) y Sentíes y Díaz-Larrea (2008) realizaron estudios moleculares en el complejo Laurencia 
en el Caribe mexicano proponiendo la combinación de Palisada poiteaui (J. V. Lamouroux) K. W. Nam var. gemmifera (Harvey) Sentíes, Fujii et Díaz-Larrea.

Con la reciente propuesta de elevación de la sección Palisadae al nivel genérico, las especies (9 en total) del género Chondrophycus registradas para el Atlántico occidental (Wynne, 2005) fueron trasladadas a Palisada por ajustarse claramente con los caracteres morfológicos, por lo que las 4 especies de Chondrophycus (Sentíes y Fujii, 2002) registradas en el Caribe mexicano pertenecen ahora a Palisada.

La combinación de $P$. perforata (Bory) K. W. Nam la estableció Nam (2007); sin embargo, desde que fue descrita por primera vez como Fucus perforatus (Bory, 1803), no se incluyeron detalles de su morfología, principalmente la reproductiva. Es por ello que en este trabajo se hace un análisis detallado de su morfología vegetativa y reproductiva (tetrasporangial), se compara y amplía su distribución y se establece este nuevo registro para la región tropical del Atlántico occidental, específicamente en el Caribe mexicano.

\section{Materiales y métodos}

Se recolectaron manualmente ejemplares de Palisada perforata (febrero 2006 y marzo 20007), en el lado oriental de isla Mujeres, Quintana Roo, México (21 $12^{\prime} 07.79^{\prime}$ ' N 86 $42^{\prime} 40.64^{\prime}$ ' O). Los ejemplares frescos y de herbario se fijaron y preservaron en formalina con agua de mar al $4 \%$. Los cortes anatómicos fueron hechos a mano usando una navaja de rasurar bajo el microscopio estereoscópico, colocando para su coloración una solución acuosa con azul de anilina al $0.5 \%$, acidificada con $\mathrm{HCl} 1 \mathrm{~N}$ (Tsuda y Abbott, 1985). Las medidas celulares se dan en largo por ancho. La figura a línea se elaboró con una cámara clara adaptada a un microscopio Nikon Eclipse E200 (Tokyo, Japón) y las fotomicrografías se tomaron utilizando una cámara digital Sony W5 (Tokio, Japón) acoplada a un microscopio Nikon.

En cada recolecta fueron examinados entre 5 y 10 ejemplares y se depositaron en el Herbario Metropolitano de la Universidad Autónoma Metropolitana-Iztapalapa (UAMIZ), México (Holmgren et al., 1990). Asimismo, se analizaron ejemplares de Brasil e islas Canarias con la intención de comparar material de otras regiones del Atlántico.

\section{Descripción}

Palisada perforata (Bory) K. W. Nam, 2007.
Algae 2007, vol. 22, p. 54 (Figs. 1-11)

Basiónimo. Fucus perforatus Bory (1803), Essai sur les iles Fortunées, vol. 1. pl 5, p. 505, Pl. 5, f. 1,

B.C. excl. A.

Laurencia perforata (Bory) Montagne (1840, p. 155)

Chondrophycus perforatus (Bory) K.W. Nam (1999, p. 463)

Localidad tipo. Tenerife, islas Canarias, España.

Morfología y anatomía vegetativa. Plantas perennes formando densos tufos o alfombras sobre las rocas, con $1-5 \mathrm{~cm}$ de altura, adheridas al sustrato por un conjunto de pies discoidales asociados a ramas laterales basales. Color marrón-violeta, consistencia cartilaginosa rígida, no adhiriéndose al papel de herbario cuando están secas. Los ejes pueden ser erectos o arqueados y decumbentes, con ramificación muy comúnmente unilateral, alterna o irregular, con 3, eventualmente con 4 órdenes de ramificación(Fig. 1). Las ramas claviformes con tricoblastos hialinos. Algunos de los ejemplares examinados muestran anastomosis en las ramas, las cuales están interconectadas por medio de pequeños discos basales secundarios que salen de los ápices de las ramas cuando convergen con otras ramas. Algunas ramas arqueadas desarrollan proliferaciones rizoidales que forman a su vez nuevos discos secundarios (Fig. 2). Ejes principales con diámetro entre 500-875 (1 040) $\mu \mathrm{m}$.

En vista superficial del talo, células epidérmicas dispuestas uniformemente en toda la planta, poligonales, isodiamétricas a ligeramente alargadas radialmente, con 31-53 x 16-42 $\mu \mathrm{m}$. Uniones secundarias entre las células epidérmicas adyacentes ausentes (Fig. 3). En corte transversal del talo, 1 o 2 capas de células epidérmicas pigmentadas y de 4 a 5 capas de células medulares (Fig. 4). Células epidérmicas externas de cuadráticas a rectangulares y dispuestas en empalizada, con 24-35 x 19-25 $\mu \mathrm{m}$ (Fig. 5). Región medular con células incoloras, de pared gruesa, redondas o ligeramente alargadas, disminuyendo gradualmente de tamaño en dirección al centro del talo. Eje vegetativo con 2 células pericentrales por segmento axial, redondeadas, un poco alargadas, con 68-112 x 51-80 $\mu \mathrm{m}$ (Fig. 6). Engrosamientos lenticulares ausentes. En corte longitudinal del talo, paredes de las células epidérmicas próximas al ápice no proyectadas.

Morfología reproductiva. Ramas tetrasporofíticas cortas, papiliformes, 0.5-2.0 mm x 930-1140 $\mu \mathrm{m}$ (Fig. 7). En el ápice de la rama fértil, cada segmento axial produce una célula pericentral adicional en la posición opuesta a las otras 2 pre-existentes. De éstas, la segunda y tercera células son fértiles, la primera permanece vegetativa (Figs. 8 y 11). Cada célula pericentral fértil produce 2 células de cobertura pre-esporangiales, la inicial del tetrasporangio y una célula 


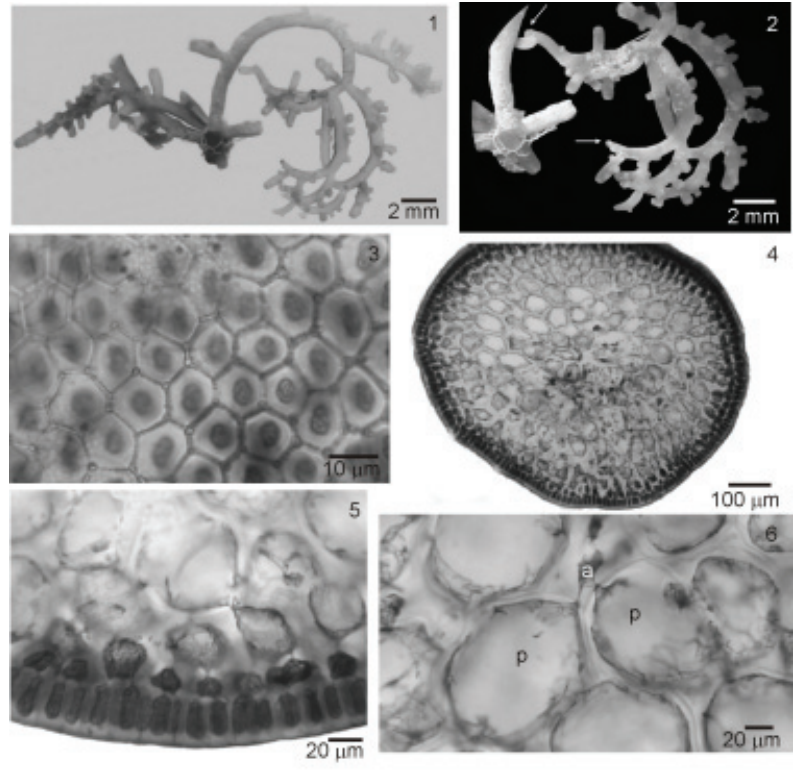

Figuras 1-6. Talo vegetativo de Palisada perforata (UAMIZ1040). 1, hábito de ejemplar del Caribe mexicano mostrando ramas arqueadas y decumbentes con ramificación unilateral. 2, Detalle de la base mostrando ramas arqueadas con fijación secundaria (flechas). 3, Vista superficial del talo mostrando ausencia de uniones secundarias entre las células epidérmicas adyacentes. 4, Corte transversal del talo. 5, Corte transversal con células epidérmicas dispuestas en empalizada. 6, Detalle de corte transversal que muestra 2 células pericentrales (p) que se originan de la célula axial (a).

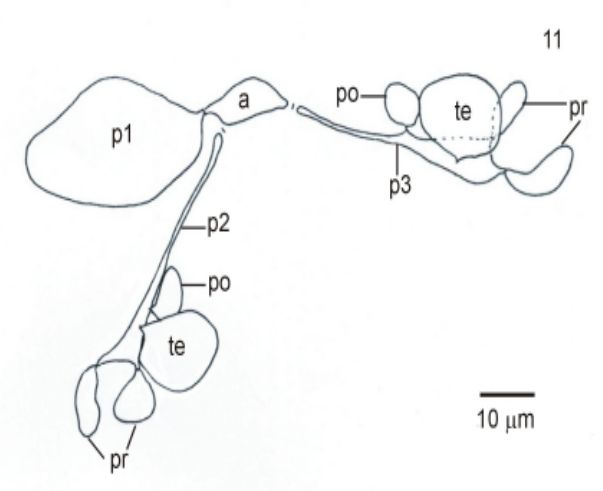

Figura 11. Esquema de corte transversal próximo al ápice de una rama tetrasporangial, mostrando célula axial (a) y 3 células pericentrales, la primera permanece vegetativa ( $\mathrm{p} 1)$, la segunda (pre-existente) y tercera (adicional) están fértiles (p2 y p3), portando cada una, la inicial del tetrasporangio (te), 2 células de cobertura pre-esporangiales (pr) y 1 post-esporangial (po).
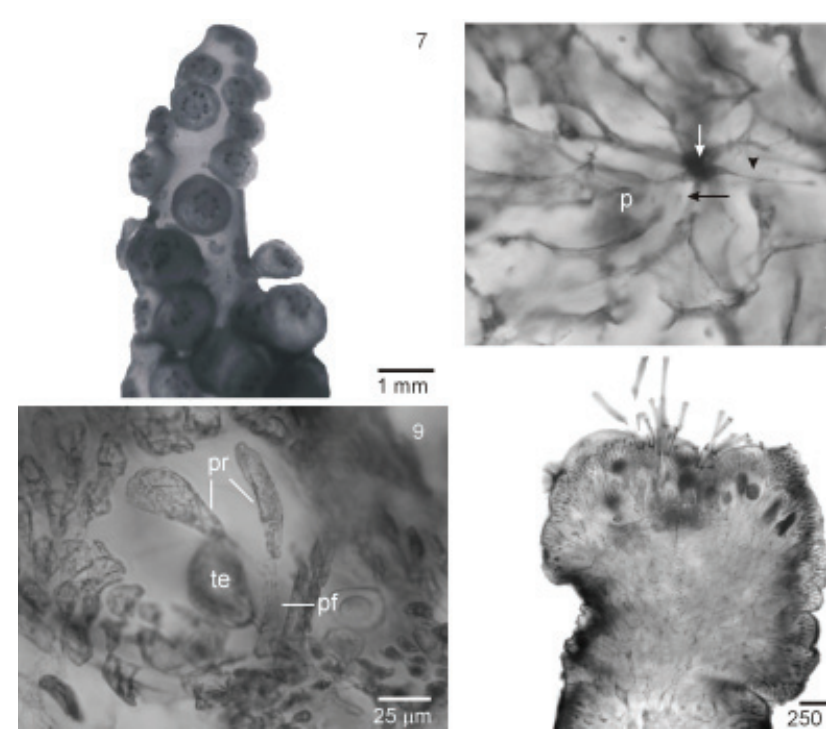

Figuras 7-10. Talo tetrasporofítico de Palisada perforata (UAMIZ-1041). 7, Rama tetrasporangial apical. 8, Corte transversal mostrando célula axial (flecha blanca) y células pericentrales (flechas negras); la primera permanece vegetativa (p); la segunda pericentral pre-existente esta fértil (flecha) y una tercera pericentral adicional fértil es formada en la posición opuesta (cabeza de flecha). 9, Corte transversal mostrando célula pericentral fértil (pf) con 2 células de cobertura pre-esporangiales (pr) y la inicial del tetrasporangio (te). 10, Corte longitudinal mostrando los tetrasporangios dispuestos en ángulo recto.

post-esporangial (Figs. 9 y 11). En vista superficial, las células de cobertura pre-esporangiales están alineadas transversalmente en relación con el eje de la rama fértil y permanecen no divididas. La célula de cobertura posesporangial se divide varias veces y contribuye al sistema de corticación del talo. Tetrasporangios tetrahédricamente divididos, 80-120 x 60-100 $\mu \mathrm{m}$, dispuestos radialmente en la rama, en arreglo del tipo ángulo recto (Figs. 7 y 10).

Hábitat. Crece formando densas alfombras en la región intermareal, sobre sustratos rocosos en zonas sometidas a periodos prolongados de desecación durante la marea baja $\mathrm{y}$ en lugares con oleaje moderado.

Material examinado. (A. Sentíes, 19.II.2006, UAMIZ1040) (A. Sentíes y MC Gil-Rodríguez, 02.III.2007, talo tetraspórico, UAMIZ-1041). Material adicional. Herbario BM (E. Bourgeau-Planta Canariensis, Tenerife, 1845. Isotypus \# 1193); Herbario de Leiden (Montagne, Canarias,1839. Herb.Lugd. bat n 941 99....104), Herbario de la Facultad de Biología de la Universidad de La Laguna, Tenerife (M. López Hernández, 12.VII.1979, Dupl. Phyc. TFC 2006; MC Gil-Rodríguez, 20.V.1984, Phyc. TFC 
2886; MC Gil-Rodríguez 8.II.2007, Dupl. Phyc. TFC 13148 ) y Herbario del Jardín Botánico de Río de Janeiro (V. Cassano, 30.XII.2005, talo tetraspórico, HRJ 10840, SP 371.774; V. Cassano, 3.VIII.2005, talo tetraspórico, HRJ 10839, SP 371.777).

Distribución mundial. Esta especie se encuentra ampliamente distribuida. Se tienen registros en los siguientes océanos y países: Atlántico oriental: islas Azores (Portugal), islas Canarias (España), Gabon, Gambia, São Tomé (África occidental). Atlántico occidental: México (Caribe). Pacífico: Japón, Vietnam, Micronesia. Índico: India, Kenya, Madagascar, Sri Lanka, Tanzania. Mediterráneo: Francia, Italia. (Guiry y Guiry, 2006).

\section{Discusión}

Palisada perforata fue establecida por Bory (1803) como Fucus perforatus, basándose en ejemplares de las islas Canarias, España, caracterizada principalmente por su crecimiento cespitoso con ejes arqueados y decumbentes y ramificación unilateral. Esta especie se incluye dentro de Palisada por la presencia de 2 células pericentrales en cada segmento axial vegetativo, tetrasporangios producidos a partir de células pericentrales particulares y eje tetrasporangial con una célula pericentral estéril y la segunda pericentral siempre fértil (Nam, 2006). Además pertenece al subgénero Palisada (Yamada) Nam, por la ausencia de uniones intercelulares secundarias entre las células epidérmicas, una célula pericentral estéril en los segmentos axiales de los tetrasporangios y el arreglo en ángulo recto de los mismos. Finalmente, se incorpora en la sección Palisadae Nam por la presencia de las células epidérmicas dispuestas en empalizada en corte transversal (Nam, 1999).

Siguiendo la descripción original de Bory (1803), complementada con los atributos analizados por Yamada (1931) y algunos caracteres morfológicos adicionales de Montagne (1840), Nam (1999), Haroun y Gil-Rodríguez (1995) y Gil-Rodríguez y Haroun (2002), los ejemplares recolectados en isla Mujeres, Caribe mexicano, fueron identificados como $P$. perforata. Estas plantas concuerdan completamente con los ejemplares de la localidad tipo. Asimismo, la comparación con los ejemplares de Brasil permitió discernir que se trata de la misma especie presente en las costas del Atlántico occidental.

La opinión de Yamada (1931) acerca de la identidad del tipo de Fucus perforatus de Bory, queda en un contexto superfluo sobre los 3 caracteres que definen esta especie (células epidérmicas alargadas radialmente y dispuestas en empalizada en corte tranversal, no proyectadas en vista superficial y la ausencia de engrosamientos lenticulares en las paredes de las células medulares). Sin embargo, concuerdan y se complementan con las descripciones posteriores y con el material de la localidad tipo recolectado por Montagne (1840), por lo que la descripción de esta especie se ha ido ampliando con caracteres morfológicos, principalmente. En este contexto, es ésta la primera vez que se describen detalladamente los atributos tetrasporangiales.

La ausencia de estructuras reproductivas gametangiales en este estudio confirma la aseveración de Haroun y Gil-Rodríguez (1995), quienes en un trabajo sobre las estrategias reproductivas de Laurencia perforata afirman que el proceso de propagación vegetativa es común en esta especie, sobre todo cuando las poblaciones se expresan en lugares expuestos al oleaje.

Palisada perforata es una especie típica de aguas tropicales y subtropicales, con amplia distribución (GilRodríguez y Afonso-Carrillo, 1980; Lawson y John, 1982; Silva et al., 1996; Wynne, 2005). Los únicos registros de este taxón en el Atlántico occidental han sido en Venezuela, como registro dudoso (Lemus, 1984) y en algunos estados de Brasil (Oliveira-Filho, 1969; Pedrini, 1980; Cocentino, 1994; Nunes, 1998). Sin embargo, la presencia de $P$. perforata en Brasil se ha considerado incierta, debido a la plasticidad de su hábito que en ocasiones hace que se confunda con P. papillosa (C. Agardh) K.W. Nam. Estas especies sólo se diferencian por estructuras externas, concretamente por la presencia de ramas arqueadas y decumbentes y por la ramificación unilateral en $P$. perforata (Fujii y Sentíes, 2005), dejando la posibilidad de que estos taxones sean coespecíficos. Estudios futuros más intensivos, contemplando datos moleculares, revelarán la posición taxonómica de esta especie.

\section{Agradecimientos}

A Kurt M. Dreckmann, por las sugerencias y críticas al artículo y al Dr. Santiago Gómez, de la Universidad Central de Venezuela, por el envío de literatura. Esta investigación se realizó con financiamiento parcial del proyecto "Macroalgas marinas tropicales" de la División de CBS-UAMI, PROMEP-SEP (P/CA-117 2006-35-52) y del Ministerio de Educación y Ciencia de España (CGL 2007-60635/BOS). MTF agradece a CNP por la beca de productividad en investigación.

\section{Literatura citada}

Ballantine, D.L. y N. E. Aponte. 1995. Laurencia coelenterata (Rhodomelaceae, Rhodophyta), a new diminutive species from the Dry Tortugas, Florida. Botanica Marina 38:417- 
421.

Bory de Saint-Vincent, JBGM. 1803. Essaui sur les îles Fortunées et l'antique Atlantide ou prècis de l'histoire général de l'Archipel des Canaries, Paris. 522 p.

Cassano, V., M.T. M. Szechy y M.T. Fujii. 2006. Laurencia caduciramulosa (Ceramiales, Rhodophyta) from Ilha Grande Bay, Rio de Janeiro, Brazil: a recent introduction into the Atlantic Ocean?. Cryptogamie, Algologie 27:265-277.

Cocentino, A.L.M. 1994. Familia Rhodomelaceae (Ceramiales Rhodophyta) na praia de Serrambi - Ipojuca - PE - Brasil. Dissertação de mestrado, Universidade Federal Rural de Pernambuco, Recife. 193 p.

Cordeiro-Marino, M., M.T. Fujii y F. Pinheiro-Joventino. 1994. Morphological and cytological studies of Laurencia furcata Cordeiro-Marino and Fujii (Rhodophyta, Rhodomelaceae): a new species from Brazil. Cryptogamic Botany 4:373380 .

Díaz-Larrea, J., A. Sentíes, M.T. Fujii, F.F. Pedroche y M. C. Oliveira. 2007. Molecular evidence for Chondrophycus poiteaui var. gemmiferus comb. et stat. nov. (Ceramiales, Rhodophyta) from the Mexican Caribbean Sea: implications for the taxonomy of the Laurencia complex. Botanica Marina 50:50-256

Fujii, T.M. y A. Sentíes, G. 2005. Taxonomia do complejo Laurencia (Rhodomelaceae, Rhodophyta) do Brasil, com ênfase nas espécies dos estados de Sâo Paulo e do Espírito Santo. In Monografías ficológicas, vol. II, A. Sentíes G. y K. M. Dreckmann, (eds.) Universidad Autónoma MetropolitanaIztapalapa/Red Latinoamericana de Botánica, México, D. F. p. 69-135.

Fujii, M. T. y M. Cordeiro-Marino. 1996. Laurencia translucida sp. nov. (Ceramiales, Rhodophyta) from Brazil. Phycologia 35:542-549.

Fujii M.T., L. Collado-Vides y M. Cordeiro-Marino. 1996. Morphological studies of Laurencia gemmifera and Laurencia poiteaui (Rhodomelaceae, Rhodophyta) from the Nichupté Lagoon System, Quintana Roo, Mexico. Botanica Marina 39:317-326.

Fujii, M. T., S. M. P. B. Guimarães, C. F. D. Gurgel y S. Fredericq. 2006. Characterization and phylogenetic affinities of the alga Chondrophycus flagelliferus (Rhodomelaceae, Ceramiales) from Brazil on the basis of morphological and molecular evidence. Phycologia 45:432-441.

Furnari G., F. Boissett., M. Cormaci y D. Serio. 2002. Characterization of Chondrophycus tenerrimus (Cremades) comb. nov. (Ceramiales, Rhodophyta), a species often misidentified as C. papillosus (C. Agardh) Garbary \& J. Harper in the Mediterranean Sea. Cryptogamie, Algologie 23:223-235.

Furnari G., M. Cormaci y D. Serio. 2001. The Laurencia complex (Rhodophyta, Rhodomelaceae) in the Mediterranean Sea: an overview. Cryptogamie, Algologie 22:331-373.

Garbary D. J. y J.T. Harper. 1998. A phylogenetic analysis of the Laurencia complex (Rhodomelaceae) of the red algae. Cryptogamie, Algologie 19:185-200.

Gil-Rodríguez, M. C. y J. Afonso-Carrillo. 1980. Catálogo de algas marinas bentónicas (Cyanophyta, Chlorophyta, Phaeophyta y Rhodophyta) para el Archipiélago Canario.
Aula de Cultura de Tenerife, Santa Cruz de Tenerife. 47 p.

Gil-Rodríguez, M. C. y R. Haroun. 2002. Vegetative and reproductive phenology of Chondrophycus perforatus and Laurencia viridis (Rhodomelaceae, Rhodophyta) in Tenerife, Canary Islands. Constancea 83.20, 15 pág. University of California Electronic Publications in Botany. (http://ucjeps. berkeley.edu/constancea/83/). Consultado: 10 agosto 2006.

Guiry, M. D. y G. M. Guiry. 2006. AlgaeBase version 4.2. Worldwide electronic publication, National University of Ireland, Galway. http://www.algabase.org (cosultado: 8 agosto 2006).

Haroun, R. J. y M. C. Gil-Rodríguez. 1995. Reproductive strategies of Laurencia perforata (Bory) Montagne (Ceramiales, Rhodomelaceae) in the Canary Islands. Nova Hedwigia 61: 269-274.

Holmgren, P. K., W. H. Holmgren y L.C. Barnett. 1990. Index herbariorum (Regnum vegetavile, vol. 120), part I :The herbaria of the world. New York Botanical Garden, New York. 693 p.

Lawson, G. W. y D. M. John. 1982. The marine algae and coastal environment of tropical West Africa. Nova Hedwigia 70:1455.

Lemus, C. A. J. 1984. Las algas marinas del golfo de Paria, Venezuela. II. Rhodophyta. Boletin del Instituto Oceanográf ico,Universidad de Oriente, Cumaná 23:55-112.

Montagne, C. 1840. Plantes cellulares. In Historie Naturalle des Iles Canaries, t. III (parte 2, secc. 4), P. B. Webb y S. Berthelot, Paris. 208 p.

Nam, K. W. 1999. Morphology of Chondrophycus undulata and C. parvipapillata and its implications for the taxonomy of the Laurencia (Ceramiales, Rhodophyta) complex. European Journal of Phycology 34:455-468.

Nam, K. W. 2006. Phylogenetic re-evaluation of the Laurencia complex (Rhodophyta) with a description of L. succulenta sp. nov. from Korea. Journal of Applied Phycology 18:679697.

Nam, K. W. 2007. Validation of the generic name Palisada (Rhodomelaceae, Rhodophyta). Algae 22:53-55.

Nam, K. W., C. A. Maggs y D. J. Garbary. 1994. Resurrection of the genus Osmundea with an emendation of the generic delineation of Laurencia (Ceramiales, Rhodophyta). Phycologia 33:384-395.

Nunes, J. M. de C. 1998. Marine benthic rhodophytes of Salvador oceanic fringe, Bahia State, Brazil. Insula (Florianópolis) 27:27-37.

Oliveira-Filho, E. C. 1969. Algas marinhas do sul do Espíritu Santo (Brasil) 1. Ceramiales. Boletim de Faculdade de Filosofia, Ciências e Letras. Universidade de São Paulo, São Paulo. 407 p.

Pedrini, A. G. 1980. Algas marinhas bentônicas da Baía de Sepetiba e arredores (Rio de Janeiro). Dissertação de Mestrado, Universidade Federal do Rio de Janeiro, Rio de Janeiro. 397 p.

Sentíes, G. A. y M. T. Fujii. 2002. El complejo Laurencia (Rhodomelaceae, Rhodophyta) en el Caribe mexicano. In Monografías Ficológicas, vol. I., A. Sentíes G. y K. M. Dreckmann (eds.). Universidad Autónoma MetropolitanaIztapalapa/ Red Latinoamericana de Botánica. México, D. F. p. 121-192. 
Sentíes, G., A., M. T. Fujii y D. Rodríguez. 2001. Laurencia venusta (Ceramiales, Rhodophyta): a new record from the Atlantic Ocean. Botanica Marina 44:95-99.

Sentíes, A. y J. Díaz-Larrea. 2008. Proposals of Palisada poiteaui var. gemmifera comb. nov. and Palisada corallopsis comb. nov. (Rhodomelaceae, Rhodophyta). Botanica Marina 51:69-70.

Silva, P., P.W. Basson y R.L. Moe. 1996. Catalogue of the Benthic Marine Algae of the Indian Ocean. vol. 79. University of California Press, Berkeley, 1259 p.

Tsuda, R.T. y I.A. Abbott. 1985. Collecting, Handling, Preservation, and Logistics. In Handbook of Phycological
Methods, vol. IV. Ecological Field Methods: Macroalgae, M. M. Littler y D. S. Littler (eds.). Cambridge University Press, Cambridge. p. 67-86

Wynne, M.J. 2005. A checklist of benthic marine algae of the tropical and subtropical western Atlantic: second revision. Nova Hedwigia 129:1-152.

Wynne, M.J. y D.L. Ballantine. 1991. Laurencia iridescens sp. nov. Rhodomelaceae, Ceramiales) from the Caribbean Sea. Phycologia 30:394-401.

Yamada, Y. 1931. Notes on Laurencia, with special refedrence to the Japanese species. University of California Publications in Botany 16:185-310. 